\title{
Experimental investigation on geometrical aspects of micro-plasma deposited tool steel for repair applications
}

\author{
S. Jhavar \\ Department of Mechanical Engineering, \\ Indian Institute of Technology, Indore MP, 452009 India \\ suyog@iiti.ac.in \\ C. P. Paul \\ Laser Materials Processing Division, \\ Raja Ramanna Centre for Advanced Technology, Indore MP, 452009 India \\ paulcp@rrat.gov.in \\ N. K. Jain \\ Department of Mechanical Engineering, \\ Indian Institute of Technology, Indore MP, 452009 India \\ nkjain@iiti.ac.in
}

Published 13 August 2014

\begin{abstract}
Recent advancement in direct material deposition processes found wide applications in rapid prototyping, manufacturing and tooling industry. Micro-plasma deposition is one of the recent developments in this domain. This paper reports the deployment of newly integrated micro-plasma deposition system for the deposition of AISI P-20 tool steel on the AISI P20 tool steel substrate. A number of test tracks for single track deposition were deposited at the various combination of processing parameters. The sets of parameters yielding good deposits were selected to deposit overlap tracks. The geometry of single and overlapped tracks was evaluated to understand the parametric dependence. The study indicates that the aspect ratio of track geometry (ratio of width to height of track) is dependent on the processing parameters and the discharge current is identified as the most dominating parameters (contribution $=44 \%$ ), followed by scan speed (contribution $=26.68 \%)$ and wire feed rate (contribution $=26.98 \%)$ with almost same effect. The microscopic study of the deposits indicates that the material deposited at the optimum processing parameters is free from surface and bulk defects. The estimated material properties are found to be at par with conventional processed material. This feasibility study proved that the micro-plasma deposition can be used for the generation of surfaces and multi-featured material deposition. It paved a way for the application of the process in die/mold repairs.
\end{abstract}

Keywords: Micro-plasma deposition; tool steel; mold/die repair.

\section{Introduction}

Molds and dies are frequently subjected to local impacts, thermal stresses and corrosion

This is an Open Access article published by World Scientific Publishing Company. It is distributed under the terms of the Creative Commons Attribution 3.0 (CC-BY) License. Further distribution of this work is permitted, provided the original work is properly cited. 
which consequently develops various defects during operation viz. plastically deformed geometries, minor cracks, deteriorated edges, heat checks, dents etc. These defects are likely to be identified during preventive maintenance of operative molds and dies. Detection and repair of these defects at initial stage can significantly improve performance, life span and profitability of molds and dies. Remanufacturing is always environmental friendly, avoids energy emission, reduces wastage, reduces redundancy in terms of stand by mold/die and saves lead time of replacement and hence becoming a common practice in industries especially for higher value components ${ }^{1}$. Ethical and moral responsibility, environmental legislation and profitability ${ }^{2}$ are identified as the three major drives that follow remanufacturing in industries. There have been a number of published reports evaluating the feasibility of developing repairing and remanufacturing procedures. Traditionally, manual multi-weld deposition was used for repair and remanufacture which was replaced by automated welding afterwards. Conventional welding resulted in low weld ability of traditional materials used for manufacturing molds and dies. These materials possess considerably high amount of carbon and alloying elements thereby making the material difficult to weld. Due to substantial metallurgical concern while repair or remanufacturing using direct metal deposition (DMD) through additive layered manufacturing (ALM) ensuring large reduction in energy consumption, environmental emission and manufacturing costs ${ }^{3}$.

Repairing of high value components developed the need for more precise 3-D deposition techniques utilizing high energy beam deposition like electron beam deposition, laser based deposition and micro plasma transferred arc ( $\mu$ PTA) deposition to be associated with accurate manipulation system viz. robotic arm, computerized numerically controlled machines etc. ensuring better and finer control. With the advancement in 3D-deposition techniques, laser based processes are found to be most successful for dies/mold repairing ${ }^{4-5}$. Studies emphasized to use laser based deposition for fine repair of dies and molds whereas conventional gas tungsten arc welding (GTAW) was used for bulk repair. Localized heating at a very narrow zone is treated as the most effective consideration while selecting among various laser based processes for deposition $^{6-8}$. A comparison between laser surfacing and submerged arc surfacing showed that laser surfacing produced favorable variation of compression residual stresses, which contributed to the extended operating life of dies and molds 9 . An application of successful deposition of AISI D2 tool steel using pulsed electron beam processing was also reported $^{10}$. Hence, laser, electron beam and electric arc are major energy sources used in ALM for repair/remanufacturing applications. Laser and electron beam are more precise energy sources than the electric arc, therefore, they are preferred for miniature sized deposition.

Poor energy conversion efficiency, causes higher energy consumption is a major drawback of high energy beam processes during fabrication of components, tooling and related applications. Use of energy efficient sources and increase in the deposition rates for ALM processes are two major issues in current ALM processes. The deposition rate achieved by electric arc based deposition processes can achieve it in the range of 50- 
$130 \mathrm{~g} / \mathrm{min}$. whereas, laser or electron beam is in the range of $2-10 \mathrm{~g} / \mathrm{min}^{11}$. Therefore, arc based deposition techniques are preferred for bulk deposition of metallic components for various ALM purposes. Micro-arc based deposition is one of the recent ALM techniques having prospective applications in repairs and fabrication at meso-scale. Successful deposition during manufacturing of three dimensional structures using micro-sized wire for miniature deposition through a micro-tungsten inert gas ( $\mu$-TIG) welding system was reported recently ${ }^{12}$. Wide applications of ALM are being used in industrial practices for die and mold repair ${ }^{13}$.

In this paper, a systematic study to indentify the optimum process parameters and characterizing its performance at various combinations of the input parameters was carried out. As aspect ratio is one of the important parameter for multi-track surfacing during repair/remanufacturing. The optimized parameters were used to develop a regression model for calculating aspect ratio at selected combinations of processing parameters. An experimental setup was developed to deposit AISI P20 tool steel wire of $300 \mu \mathrm{m}$ diameter on the substrate of the same material. Experiments were conducted to indentify the process parameters for producing regular and smooth single track geometry.

\section{Material and Methods}

AISI P20 tool steel is one of the widely used materials containing Cr- Mo, and capable of withstanding forces used in Zinc die-casting and plastic molding. It can be polished to extremely high surface finish and hence, it is best suitable for medium and large size moulds, extrusion dies, injection moulds, press-brake dies, aluminum die casting, and forming tools. In the present study, pre-hardened AISI P-20 tool steel samples of size 100 $\mathrm{mm} \times 180 \mathrm{~mm} \times 9 \mathrm{~mm}$ was used as substrate. The samples were cut, machined, shot blasted and cleaned with acetone before being used for deposition. Deposited material was in the form of fine wire of size $300 \mu \mathrm{m}$. The chemical composition of both substrate and deposited wire is presented in Table 1.

Table 1. Chemical composition of substrate and deposited material.

\begin{tabular}{llllllll}
\hline Elements & $\mathrm{C}$ & $\mathrm{Mn}$ & $\mathrm{Cr}$ & $\mathrm{Mo}$ & $\mathrm{Si}$ & $\mathrm{Cu}$ & $\mathrm{Ni}$ \\
\hline Substrate & 0.40 & 1.5 & 1.9 & 0.2 & 0.1 & - & - \\
Deposited Wire & 0.1 & 0.97 & 1.9 & 1.03 & 0.54 & 0.03 & 0.03 \\
\hline
\end{tabular}

$\mu$ PTA deposition system is presented in schematic diagram shown in Fig. 1. The nozzle of $\mu$ PTA system was suitably modified for feeding the wire to the melt-pool. Argon gas (Purity $>99.995 \%$ ) was used for the above experiments for plasma generation as well as shielding purposes. The $\mu$ PTA has maximum current capacity of $18 \mathrm{~A}$ and could be varied in a step of $0.1 \mathrm{~A}$. This workstation has a travel of $300 \mathrm{~mm}$ and $160 \mathrm{~mm}$ in $\mathrm{X}$ and Y direction, respectively. The maximum speed of travel used in the study was 100 $\mathrm{mm} / \mathrm{min}$. The wire-feeder has a capability of feeding wire in the range 850 to 1700 $\mathrm{mm} / \mathrm{min}$. Comprehensive experiments were planned in two steps (a) pilot 
experimentation and (b) final experimentation. The three main parameters (input current, machine travel speed and wire feed rate) were identified as significant parameters for depositing single track over AISI P20 tool steel substrate through pilot experiments.

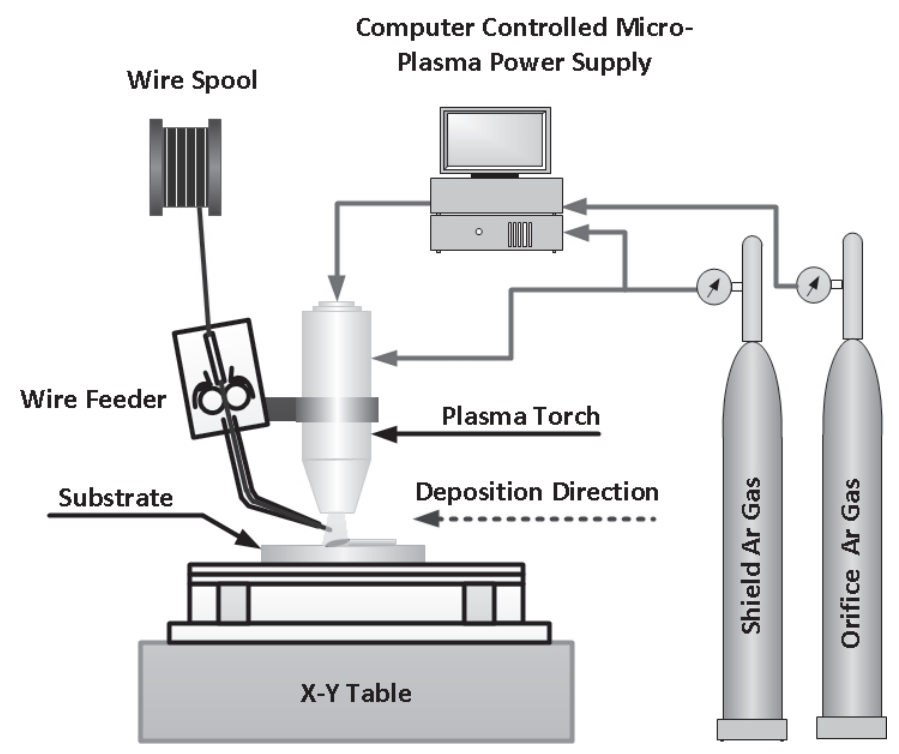

Fig. 1. Experimental Apparatus.

Table 2 presents the parameters and their ranges used in the study. Other parameters, viz. torch stand-off distance, plasma orifices gas flow rate and shield gas flow rate, are important during the transferring of plasma. The values of parameters i.e. torch standoff, plasma orifices gas flow rate and shielding gas flow rate were $5 \mathrm{~mm}, 0.4 \mathrm{Nl} / \mathrm{min}$ and 5 $\mathrm{Nl} / \mathrm{min}$, respectively and were kept constant throughout the study. A total of forty five experiments were performed as shown in Table 3. These deposited tracks were cut transverse to the direction of deposition and the samples were prepared using standard metallographic procedure for optical microscopy. Standard optical microscope was used to measure track geometry (height and width).

Table 2. Processing parameters and their ranges.

\begin{tabular}{lll}
\hline Parameters & Unit & Value \\
\hline Plasma Power & $\mathrm{W}$ & $350,400,450$ \\
Machine travel speed & $\mathrm{mm} / \mathrm{min}$ & $40,50,63,80,100$ \\
Wire feed rate & $\mathrm{mm} / \mathrm{min}$ & $850,1275,1700$ \\
Wire feeding angle & Degree & 44 \\
\hline
\end{tabular}


Geometrical aspects of $\mu$-plasma deposited tool steel for repair applications

Table 3. Sets of processing parameters and their respective outcomes.

\begin{tabular}{|c|c|c|c|c|c|c|}
\hline $\begin{array}{c}\text { Track } \\
\text { ID }\end{array}$ & $\begin{array}{c}\text { Plasma } \\
\text { Power ' } P \text { ' } \\
\text { (W) }\end{array}$ & $\begin{array}{c}\text { Travel } \\
\text { speed ' } v \text { ' } \\
(\mathrm{mm} / \mathrm{min})\end{array}$ & $\begin{array}{c}\text { Wire Feed } \\
\text { Rate 'f' } \\
(\mathrm{mm} / \mathrm{min})\end{array}$ & $\begin{array}{l}\text { Width } \\
(\mathrm{mm})\end{array}$ & $\begin{array}{l}\text { Height } \\
(\mathrm{mm})\end{array}$ & $\begin{array}{c}\text { Aspect } \\
\text { Ratio }\end{array}$ \\
\hline 1 & 350 & 40 & 1700 & 1.9 & 1.8 & 1.06 \\
\hline 2 & 350 & 50 & 1700 & 1.8 & 1.6 & 1.13 \\
\hline 3 & 350 & 63 & 1700 & 1.5 & 1.3 & 1.15 \\
\hline 4 & 350 & 80 & 1700 & 1.4 & 1.2 & 1.17 \\
\hline 5 & 350 & 100 & 1700 & 1.2 & 1 & 1.20 \\
\hline 6 & 350 & 40 & 1275 & 1.6 & 1.4 & 1.14 \\
\hline 7 & 350 & 50 & 1275 & 1.5 & 1.3 & 1.15 \\
\hline 8 & 350 & 63 & 1275 & 1.4 & 1.2 & 1.17 \\
\hline 9 & 350 & 80 & 1275 & 1.2 & 1 & 1.20 \\
\hline 10 & 350 & 100 & 1275 & 1.1 & 0.8 & 1.38 \\
\hline 11 & 350 & 40 & 850 & 1.5 & 1.2 & 1.25 \\
\hline 12 & 350 & 50 & 850 & 1.3 & 1 & 1.30 \\
\hline 13 & 350 & 63 & 850 & 1.3 & 0.8 & 1.63 \\
\hline 14 & 350 & 80 & 850 & 1.4 & 0.7 & 2.00 \\
\hline 15 & 350 & 100 & 850 & 1.5 & 0.6 & 2.50 \\
\hline 16 & 400 & 40 & 1700 & 2 & 1.6 & 1.25 \\
\hline 17 & 400 & 50 & 1700 & 2 & 1.4 & 1.43 \\
\hline 18 & 400 & 63 & 1700 & 1.9 & 1.1 & 1.73 \\
\hline 19 & 400 & 80 & 1700 & 1.8 & 0.9 & 2.00 \\
\hline 20 & 400 & 100 & 1700 & 1.9 & 0.8 & 2.38 \\
\hline 21 & 400 & 40 & 1275 & 1.9 & 1.4 & 1.36 \\
\hline 22 & 400 & 50 & 1275 & 1.8 & 1.2 & 1.50 \\
\hline 23 & 400 & 63 & 1275 & 1.8 & 1 & 1.80 \\
\hline 24 & 400 & 80 & 1275 & 1.9 & 0.9 & 2.11 \\
\hline 25 & 400 & 100 & 1275 & 2 & 0.8 & 2.50 \\
\hline 26 & 400 & 40 & 850 & 1.8 & 0.9 & 2.00 \\
\hline 27 & 400 & 50 & 850 & 1.7 & 0.8 & 2.13 \\
\hline 28 & 400 & 63 & 850 & 1.6 & 0.7 & 2.29 \\
\hline 29 & 400 & 80 & 850 & 1.8 & 0.6 & 3.00 \\
\hline 30 & 400 & 100 & 850 & 1.9 & 0.5 & 3.80 \\
\hline 31 & 450 & 40 & 1700 & 2.1 & 1.5 & 1.40 \\
\hline 32 & 450 & 50 & 1700 & 2.1 & 1.3 & 1.62 \\
\hline 33 & 450 & 63 & 1700 & 2 & 1 & 2.00 \\
\hline 34 & 450 & 80 & 1700 & 1.9 & 0.9 & 2.11 \\
\hline 35 & 450 & 100 & 1700 & 2.1 & 0.8 & 2.63 \\
\hline 36 & 450 & 40 & 1275 & 2.2 & 1.3 & 1.69 \\
\hline 37 & 450 & 50 & 1275 & 2.1 & 1.1 & 1.91 \\
\hline 38 & 450 & 63 & 1275 & 1.9 & 0.9 & 2.11 \\
\hline 39 & 450 & 80 & 1275 & 1.7 & 0.7 & 2.43 \\
\hline 40 & 450 & 100 & 1275 & 2 & 0.6 & 3.33 \\
\hline 41 & 450 & 40 & 850 & 1.9 & 0.9 & 2.11 \\
\hline 42 & 450 & 50 & 850 & 2.1 & 0.8 & 2.63 \\
\hline 43 & 450 & 63 & 850 & 1.8 & 0.6 & 3.00 \\
\hline 44 & 450 & 80 & 850 & 2 & 0.4 & 5.00 \\
\hline 45 & 450 & 100 & 850 & 0.6 & 0.1 & 6.00 \\
\hline
\end{tabular}




\section{Results and Discussion}

\subsection{Combined effect of processing variables on track geometry}

Use of higher wire feed rate and lower plasma power consequences in insufficient melting of the filler material leading to a lower bonding between the substrate and the filler material. This may also result collision in extreme cases. The collision produced during deposition may deflect delivery of the filler wire, offsetting it from the center position. Center offsetting may cause wrong wire positioning and irregular deposits. Conversely, it was observed that use of higher values of plasma power and lower wire feed rate resulted in evaporation of the deposited material leading to discontinuity, building cavities, over-melting of the base and filler material and either no or very litter deposition. As wire positioning to plasma delivery center is very important in order to utilize the maximum plasma energy and produce sound deposition. It was thereby noted that, there is a balance of plasma power and wire feed rate required for sound deposition.

\subsection{Effects of plasma power, wire feed rate and travel speed on the height (h) and width (w) of the deposited track profile}

The analysis to the results shows that process response indicated the most significant process variable affecting the deposited track height and width. As depicted from Fig. 2 that a change in plasma power had little or no effect on the height of the deposited track, whereas the width of the deposited track increases with an increase in plasma power. An increase in plasma power results in a direct increase in the energy input into the substrate leading to an increase in the width of the melt pool on the surface of the substrate.

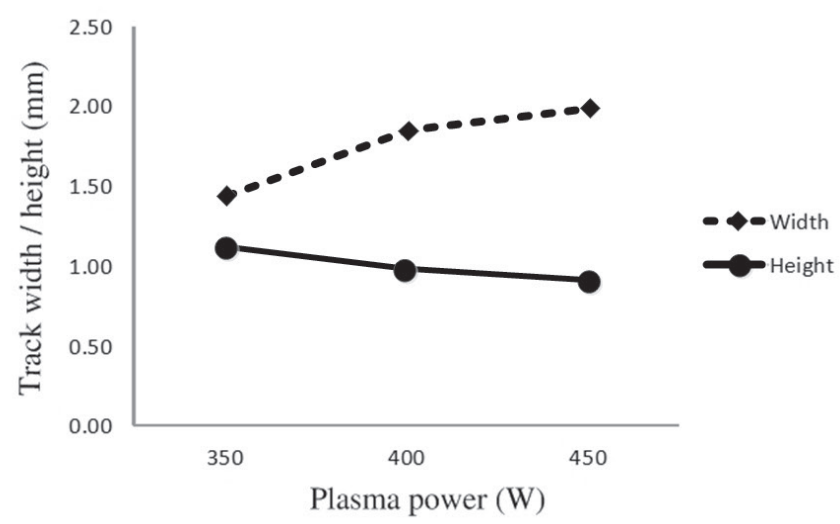

Fig. 2. Effect of Plasma power over width and height of deposited track. 
Height of the deposited track increased with an increase in the wire feed rate. The increase could be directly attributed to an increase in the amount of wire being deposited per unit length with an increase in wire feed rate. Also, the width of the deposited track increased with an increase in the wire feed rate. Inspection of the deposited track cross section revealed that when the deposited layer spread too far beyond the melt - substrate interface the ends of the deposited track often did not form a proper metallurgical bond with the substrate.

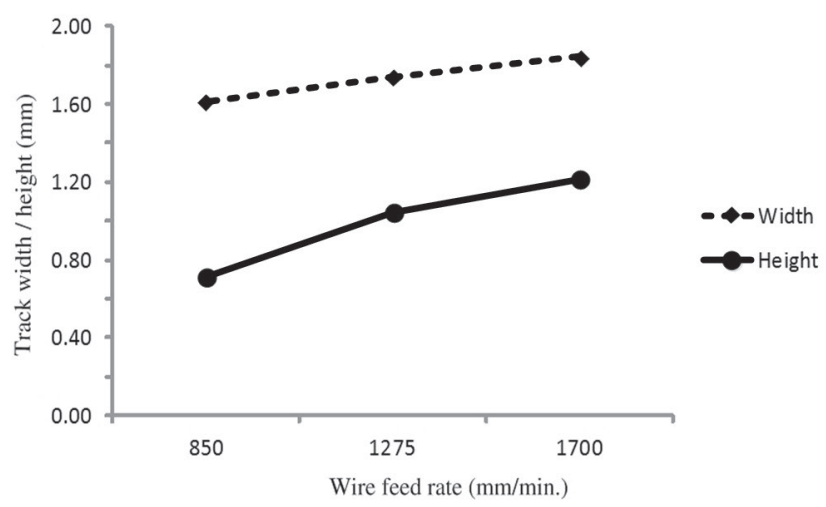

Fig. 3. Effect of wire feed rate over width and height of deposited track.

The height of the deposited track decreased with an increase in the machine travel speed. The decrease could be attributed to a decrease in the amount of wire delivered per unit length with an increase in the machine travel speed. Subsequently, an increase in the machine travel speed has an inverse effect on the width of the deposited track when compared to plasma power. The width of the deposited track decreased almost linearly with an increase in the machine travel speed. The decrease in the width of the deposited track could be attributed to a reduction in the interaction time between the plasma and the substrate, and thus also the energy input per unit length.

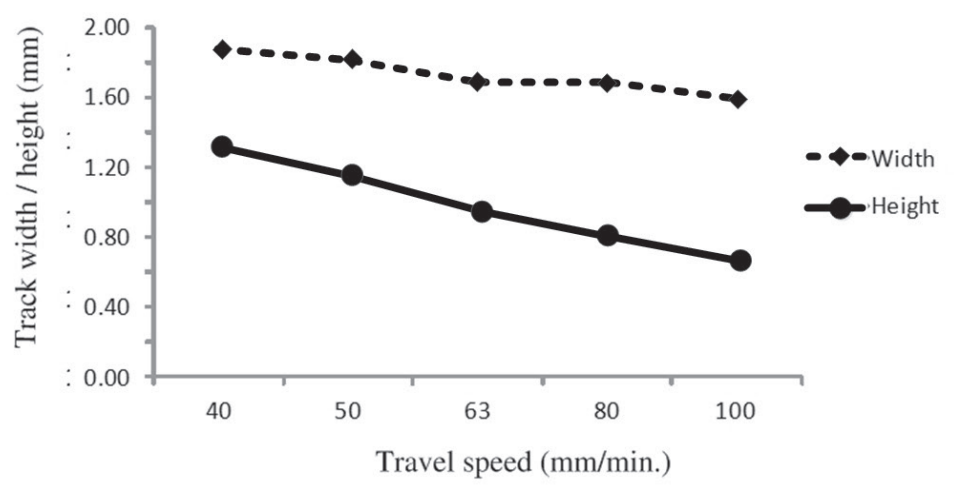

Fig. 4. Effect of travel speed over width and height of deposited track. 
It can therefore be interpreted from the above analysis that the most significant process variable affecting the deposited track height is wire feed rate, followed by machine travel speed. On the other, all the three main process variables are influenced the width of the deposited track. A change in plasma power seems to have the most significant effect on the width of the deposited track, followed by the machine travel speed and wire feed rate.

\subsection{Effect of processing variables on aspect ratio}

Aspect ratio can be calculated from the measured width and height and is a ratio of width to height of the deposited track. Favorable ranges of aspect ratios are essential in order to avoid inter-run porosity in these overlapping tracks ${ }^{14}$. Hence, on the basis of experiments performed it is important to model aspect ratio and contribution of each processing parameters. Through visual examination, nine out of forty five combinations of plasma power, wire feed rate and travel speed, were selected producing smooth and regular deposition. Table 4 presents the selected parameters used to model aspect ratio. It was also revealed through experimental results that good deposition is achieved when the aspect ratio ranges between 1.3 and 4 .

Table 4. Selected sets of processing parameters and their respective aspect ratio.

\begin{tabular}{ccccc}
\hline Exp. No. & $\begin{array}{c}\text { Plasma } \\
\text { power } \\
(\mathrm{W})\end{array}$ & $\begin{array}{c}\text { Travel speed of } \\
\text { worktable } \\
(\mathrm{mm} / \mathrm{min} .)\end{array}$ & $\begin{array}{c}\text { Wire feed rate } \\
(\mathrm{mm} / \mathrm{min} .)\end{array}$ & Aspect ratio \\
\hline 12 & 350 & 50 & 850 & 1.30 \\
13 & 350 & 63 & 850 & 1.63 \\
14 & 350 & 80 & 850 & 2.00 \\
27 & 400 & 50 & 850 & 2.13 \\
28 & 400 & 63 & 850 & 2.29 \\
29 & 400 & 80 & 850 & 3.00 \\
38 & 450 & 63 & 1275 & 2.11 \\
39 & 450 & 80 & 1275 & 2.43 \\
40 & 450 & 100 & 1275 & 3.33 \\
\hline
\end{tabular}

Based on the selected nine parameters generating smooth and regular tracks were used to develop a mathematical relationship shown in equation (1) in order to predict aspect ratio. The aspect ratio of track geometry is dependent on the processing parameters and the discharge current is identified as the most dominating parameters (contribution = $44 \%$ ), followed by scan speed (contribution $=26.68 \%$ ) and wire feed rate (contribution $=$ $26.98 \%$ ) with almost same effect.

$$
\mathrm{AR}=-4.03+0.470 \mathrm{P}+0.020 \mathrm{v}-0.003 \mathrm{f}
$$

\section{Conclusion}

This paper reported the deployment of newly integrated micro-plasma deposition system for the deposition of AISI P-20 tool steel on the same substrate. A systematic study was 
carried out to identify the optimum process parameters and characterizing its performance at various combinations. The study indicates that the aspect ratio of track geometry (ratio of width to height of track) is dependent on the processing parameters and the discharge current is identified as the most dominating parameters (contribution $=$ $44 \%$ ), followed by scan speed (contribution $=26.68 \%$ ) and wire feed rate (contribution $=$ $26.98 \%$ ) with almost same effect. The microscopic study of the deposits indicates that the material deposited at the optimum processing parameters is free from surface and bulk defects.

\section{References}

1. S. Thompson, Handbook of mold tool and die repair welding, $1^{\text {st }}$ edn. (William Andrew Publishing. Cambridge, UK, 1999).

2. Seitz, M. A., Journal of Cleaner Production 15, 1147-1157 (2007).

3. W. R. Morrow, H. Qi, I. Kim, J. Mazumder, S. J. Skerlos, Journal of Cleaner Production 15, 932-943 (2007).

4. W. T. Preciado, C. E. N. Bohorquez, Journal of Materials Processing Technology 179, 244250 (2006).

5. A. Kosnik, J. Tuek, L. Kosec, T. Muhi, METABK 50-4, 231-234 (2011).

6. L.P. Borrego, J.T.B. Pires, J.M. Costa, J.M. Ferreira, Engineering Failure Analysis 16, 596607 (2009).

7. J. S. Lim, K. L. Ng, K. M. Teh, SIMTech technical reports 9-3, 142-147 (2008).

8. J. Leunda, C. Soriano, C. Sanz, V. G. Navas, Physics Procedia 12, 345-352 (2011).

9. J. Grum and J. M. Slabe, Applied Surface Science 208-209, 424-431 (2003).

10. J.X. Zou, T. Grosdidier, K.M. Zhang, C. Dong, Applied Surface Science 255, 4758-4764 (2009).

11. S. Suryakumar, K.P. Karunakaran, A. Bernard, U. Chandrasekhar, N. Raghavender, D. Sharma, Computer-Aided Design 43, 331-344 ( 2011).

12. T. Horii, S. Kirihara, Y. Miyamoto, Materials \& Design 30, 1093-1097 (2009).

13. S. Jhavar, C.P. Paul, N.K. Jain, Causes of failure and repairing options for dies and molds: a review, Engineering Failure Analysis 34, 519-535 (2013).

14. C.P. Paul, S.K. Mishra, A. Kumar, L.M. Kukreja, Surface and Coating Technology 224, 1828 (2013). 